\title{
Modified National Early Warning Score as Early Predictor of Outcome in COVID-19 Pandemic
}

\author{
Fabio Tagliabue ${ }^{1}$. Daniele Schena ${ }^{3} \cdot$ Luca Galassi $^{1}$ (D) Matteo Magni ${ }^{1} \cdot$ Guglielmo Guerrazzi ${ }^{2} \cdot$ Andrea Acerbis $^{1}$. \\ Christina Rinallo ${ }^{3}$ - Daniel Longhi ${ }^{4}$ - Alberto Ronzani $^{5}$ - Pierpaolo Mariani ${ }^{1}$
}

Accepted: 9 June 2021 / Published online: 18 June 2021

(C) The Author(s), under exclusive licence to Springer Nature Switzerland AG 2021

\begin{abstract}
COVID-19 represented an important challenge to the Italian healthcare system (IHCS). Our main aim was to obtain evidence to support the use of modified national early warning score (m-NEWS) as an interdisciplinary, common, and universal scoring scale to quickly recognize patients with a risk of clinical deterioration before admission and during hospitalization. As a secondary goal, we tried to find a score threshold that can trigger patients' immediate medical review as a part of an optimal triaging protocol for an emergency setting where healthcare resources are overloaded. We performed a retrospective observational study. We included in our study all patients treated for COVID-19 infection in surgical departments between 01 March 2020 and 16 April 2020. Patients with negative test results for SARS-COV-2 were excluded. m-NEWS was obtained twice a day. Patients' mNEWS were analyzed in order to verify the correlation between m-NEWS (at admission and m-NEWS variation $24 \mathrm{~h}$ after admission) and outcome (positive outcome-survival, negative outcome-death, or intensive care unit (ICU) transfer). We included a population-based sample of 225 SARS-COV-2-infected patients. Overall, the average age at hospitalization was 71 (ranging from 40 to 95$) .144$ (64\%) patients were males and 81 (36\%) females. m-NEWS values lower or equal to 7 were associated with the majority of the "recovered" population (100/132 75.75\%) and at the same time with the minority of the "non-recovered" population $(25 / 9326.88 \%)$. For our sample, age is statistically correlated to the outcome but a triage protocol based solely on this variable is less effective than m-NEWS, which showed to be a reliable and easy-to-use score for first patient evaluation. Our observations pave the way towards further studies aiming at optimizing territorial and community healthcare management protocols.
\end{abstract}

Keywords EWS $\cdot$ COVID-19 $\cdot$ Score $\cdot$ Respiratory failure

Fabio Tagliabue and Daniele Schena contributed equally to this work.

Luca Galassi

lucagalassimd@gmail.com

1 ASST Bergamo Est, P.O. Pesenti Fenaroli, General Surgery Unit, Alzano Lombardo, Bergamo, Italy

2 University of Milan, Via Festa del Perdono 7, 20122 Milan, Italy

3 ASST Bergamo Est, P.O. Pesenti Fenaroli, Orthopaedics and Traumatology Unit, Alzano Lombardo, Bergamo, Italy

4 Polytechnic University of Milan, Piazza Leonardo da Vinci, 32, 20133 Milan, Italy

5 VTT Technical Research Centre of Finland Ltd., 02150 Espoo, Finland

\section{Introduction}

COVID-19 outbreak represented an important challenge for the Italian healthcare system (IHCS). Concerns regarding the capacity of the IHCS to respond to the needs of all infected patients came to light. Lots of COVID-19-infected patients needed to be treated in hospitals, and some of them required intensive care unit assistance. Alzano Lombardo Hospital is located in one of the most severely hit areas (BergamoLombardy). Due to the unavailability of ICU in our hospital and to the insufficient number of beds in the internal medicine departments, patients had to be redistributed in other nonspecialized wards such as obstetrics and gynecology, orthopedics, or general surgery units or in other hospitals whenever ICU was needed. Bergamo hinterland ICUs were under pressure, and lots of new beds had to be arranged in a short time to provide care for every infected patient (ICUs in Lombardy: 
capacity increased from 859 to 1600 ). Moreover, during the pandemic, graduated medical students and military physicians were called to help specialists in managing SARS-COV-2 patients [1]. With numerous differently specialized physicians working together, it appeared very useful to develop standardized clinical strategies in order to maximize medical efforts and find the most effective way to treat COVID-19. With the collapse of the majority of hospitals' emergency departments, ordinary internal medicine wards, and critical care units, peripheral hospitals were tasked with the role of being an effective "check-point" during the first triage. The fundamental responsibility was to sort COVID-19-infected patients in the correct facilities, basing the sorting process on the actual needs of the patients. Specifically, the key aspect was to detect whether high- or low-intensity care was required [2].

In this context, we consider a simple scoring card that can identify patients with high risk of clinical evolution. The score could help different specialists to give the optimal care in the exact moment it is needed and to wisely use the few available resources [3] reserving the ICU beds only for the most critical patients [4].

Early warning score (EWS) [5], also known as the "track and trigger" system, was developed to allow early recognition of the evolution of hospitalized patients. The concept of the EWS was introduced in 1997 [6]. It is a compound numerical score, weighted by the severity of basic physiological variables, such as systolic blood pressure (SBP), heart rate (HR), respiratory rate (RR), oxygen saturation $\left(\mathrm{SpO}_{2}\right)$, temperature, mental state, and supplemental oxygenation. EWS is an objective score; it does not depend on the operator. Each item is scored 0-3 and combined to give an overall score. This score is easily calculated during the routine bedside observations, and it is considered helpful in recognizing patients who are exhibiting signs of acute deterioration. Patients are divided into five "warning levels" based on the score: white $(0$ point), yellow (1-4), orange (5-6), and red and black ( $\geq 7$ ), with correspondingly increasing risk categories, clinical responses, and solutions. In 2012, the Royal College of Physicians proposed a modified version of EWS scale called NEWS. It differs from the previous scale because it introduces the state of consciousness evaluation with a score from 0 to 3 points. Eventually, in 2017, the revised version of NEWS was released (NEWS2), introducing for the first time different oxygen saturation levels for patients with hypercapnic respiratory failure who have lower clinically recommended oxygen saturation (88-92\%).

Since their release, NEWS and NEWS2 scales have been implemented both in acute and territorial care (such as ambulance services). It showed to be a reliable and sensible system to monitor patient's conditions and detect any deterioration, allowing professionals to administer clinical care in a timely fashion [7].

Both NEWS and NEWS2 scales have been approved and tested also to be used in intensive care units too, such as to monitor patients with incoming sepsis $[8,9]$. Criticism showed up regarding predictivity of EWS due to small sample size reported in literature and the unavailability of data during patient hospitalization [10].

Moreover, the Chinese experience in COVID-19 management has suggested the use of a modified version of NEWS [11] (m-NEWS): it assigns 3 more points when a patient's age is over or equal to 65 (image 1). Age parameter, as already demonstrated in literature [12], is a risk factor for survival on its own whenever considering COVID-19-infected patients $[13,14]$ Table 1.

\section{Objectives}

During the SARS-COV-2 pandemic, the rising pressure on emergency departments all over Italy forced the peripheric hospitals to triage an enormous number of infected patients in a short timeframe to decide if a patient could be treated at home, in a low-intensity department, or in ICU. In literature, there are few articles about the application of m-NEWS in the specific context of COVID-19: the only paper describing the use of m-NEWS in COVID-19 [11] is a descriptive account, inviting further evidence gathering. Our first aim is to find any correlation between m-NEWS on admission and clinical outcome in order to quickly identify patients with a high risk of clinical deterioration to give them the best treatment at the right time (both deceased patients and patients transferred in ICU have been considered as "negative outcome" from the point of view of emergency management). As a secondary goal, we will try to find a score threshold that can trigger patients' immediate medical review as a part of an optimal triaging protocol for an emergency setting where healthcare resources are overloaded.

\section{Materials and Methods}

The retrospective observational study (approved by the local ethical committee) was performed in Alzano Lombardo Hospital. We included in our study all patients (225) treated for COVID-19 infection in surgical departments (orthopedic surgery, general surgery, and gynecology) between 01 March 2020 and 16 April 2020. None of the patients underwent surgical operations during the hospitalization, and none had surgical-related pathologies. All surgical area beds had been temporarily repurposed for COVID-19-related admissions. We excluded patients with negative test results for SARS-COV-2. Each patient was hospitalized after a first evaluation in the emergency room, where chest computed tomography scan or chest radiography, ECG, COVID-19 rhinopharyngeal swab, and routine blood examination (blood count, coagulation profile, myocardial exams, renal and liver function, PCR, lactate dehydrogenase, and electrolytes) were obtained. As soon as COVID-19 infection was 
Table 1 Modified national early earning score (m-NEWS) [11]

\begin{tabular}{|c|c|c|c|c|c|c|c|}
\hline Physiological parameters & 3 & 2 & 1 & 0 & 1 & 2 & 3 \\
\hline Age & & & & $<65$ & & & $\geq 65$ \\
\hline Respiration rate & $\leq 8$ & & $9-11$ & $12-20$ & & & $\geq 25$ \\
\hline $\mathrm{SpO}_{2} \%$ & $\leq 91$ & $92-93$ & $94-95$ & $\geq 96$ & & & \\
\hline Supplemental $\mathrm{O}_{2}$ & & Yes & & No & & & \\
\hline Temperature & $\leq 35.0$ & & $35.1-36.0$ & $36.1-38.0$ & $38.1-39$ & $\geq 39.1$ & \\
\hline Systolic BP & $\leq 90$ & $91-100$ & $101-110$ & $111-219$ & & & $\geq 220$ \\
\hline Heart rate & $\leq 40$ & & $41-50$ & $51-90$ & $91-110$ & $111-130$ & $\geq 131$ \\
\hline Consciousness level & & & & Conscious & & & Unconscious \\
\hline
\end{tabular}

suspected, following the standing SIAARTI (Società Italiana di Anestesia Analgesia Rianimazione e Terapia Intensiva) [15] and SIMIT (Società Italiana di Malattie Infettive e Tropicali) [16] guidelines, a standardized medical therapy with lopinavir/ritonavir, hydroxychloroquine, subcutaneous therapeutic EBPM, azithromycin, and ceftriaxone was started. Oxygen therapy was administered with different concentrations in relation to $\mathrm{O}_{2}$ blood saturation. The patients were constantly evaluated by medical personnel (i.e., anesthesiologists assisted by nurses trained as first responders) for possible invasive treatment (such as orotracheal intubation) and transfer to an ICU-equipped hospital. The majority of patients were admitted to our facility for SARS-COV-2related respiratory failure (95\%). All demographic data, including age, gender, comorbidities (cardiopathic disease, arterial hypertension, diabetes type II, COPD, and tumors), and date of symptoms onset, were collected. Limited to data gathering, the schedule for evaluation was at the moment of admission and, afterwards, throughout all hospitalization, two times per day. In parallel, supplementary clinical care was administered when patients were judged at high risk of deterioration. Clinical parameters were measured by qualified nurses; scores were then calculated by the medical team and inserted in patients' clinical diaries. After discharge, COVID-19 patients' m-NEWS were analyzed in order to verify the correlation between outcome (survival vs. death/ICU transfer) and

- Age

- m-NEWS at admission

- $\mathrm{m}-\mathrm{NEWS}$ variation $24 \mathrm{~h}$ after admission

\section{Results}

The extensive data collected on a sizable population treated with uniform methodology allows us to evaluate and improve the triaging of COVID-19 patients based on numerical, a posteriori analysis. Dataset manipulation, statistical analysis, and plotting were performed with a Python-based open-source software toolkit [17-21]. For each patient, the m-NEWS at hospitalization and its $24 \mathrm{~h}$ variation were estimated from time-series data interpolated with a cubic spline function. These quantities, together with patient age, were considered for statistical analysis. Probability density functions and cumulative distribution functions were estimated using Gaussian kernels with bandwidth determined according to Silverman's method [22].

Our cohort of hospitalized patients, from 1 March 2020 to 16 April 2020, for COVID-19-related respiratory failure was made of 225 patients: 144 (64\%) males and 81 (36\%) females with an average age of 71 . Patients under 65 years were 64 (28.44\%), 65-80 years were 97 (43.11\%), and over 80 patients were $64(28.84 \%)$.

Of the 225 patients, $130(57.77 \%)$ have recovered, 77 $(34.22 \%)$ died, $18(8 \%)$ were transferred in ICU. Analyzing their clinical conditions, all patients that needed ICU were intubated and then transferred in other hospitals scattered throughout all the Italian territory due to the absence of ICU in our hospital. We unfortunately were not able to collect data about their clinical course.

We noticed 43 patients (19\%) without any comorbidities (death rate: 10\%), $71(32 \%)$ with one comorbidity (death rate: $26 \%$ ), and $111(49 \%)$ with two or more comorbidities (death rate 64\%). The most common comorbidity was hypertension $121(53,77 \%)$, followed by heart diseases $62(27.55 \%)$, active neoplasm or neoplasia in remission 55 (24.44\%), diabetes mellitus 49 (21.77\%), and COPD $18(8.00 \%)$. We noticed that $39 \%(47 / 121)$ of patients with high blood pressure and $52 \%$ (32/62) of patients with heart disease died. We also observed $56 \%(10 / 18)$ deceased patients affected by COPD Table 2 .

The statistical sampling (Fig. 1) is further differentiated in terms of clinical outcome and biological sex. In the figure, dashed lines indicate population quartiles, and the distribution amplitude is proportional to the observation count of each case. From the data shown, it can be seen that the m-NEWS distribution appears overall more symmetric in male/female bias, compared to the age variable.

As expected, age is correlated with outcome; the relatively high superposition of the age distribution density curves for 
Table 2 Demographics of patient population

\begin{tabular}{ll}
\hline Total patients & 225 \\
\hline M:F & $144(64 \%): 81(36 \%)$ \\
Average age & 71 \\
Age distribution & Age under $65: 64(28.44 \%)$ \\
& Age $65-80: 97(43.11 \%)$ \\
& Age over $80: 64(28.44 \%)$ \\
No. comorbidities & None: $43(19 \%)$ \\
& One: $71(32 \%)$ \\
Outcome & Two or more: $111(49 \%)$ \\
& Recovered $130(58 \%)$ \\
& ICU $18(8 \%)$ \\
Death rate related to comorbidities & Dead $77(34 \%)$ \\
& $10 \%$ none comorbidity \\
& $26 \%$ one comorbidity \\
& $64 \%$ two or more comorbidities \\
\hline
\end{tabular}

the three outcome groups (Fig. 2a) suggests that a triage protocol based solely on this variable would be far from optimal. In comparison, the higher segregation found between the distribution density curves estimated for the m-NEWS at the time of admission (Fig. 2b) suggests the adoption of this latter quantity as a more solid foundation of a triage protocol. We notice pronounced positive correlation between age and $\mathrm{m}$ NEWS at the time of admission (Fig. 2d), at least partially due to the inclusion of the age-related modifier in the latter. Finally, the initial $24 \mathrm{~h}$ difference in m-NEWS appears poorly correlated to outcome (Fig. 2c), although we notice a negative correlation with the m-NEWS at the time of admission (Fig.

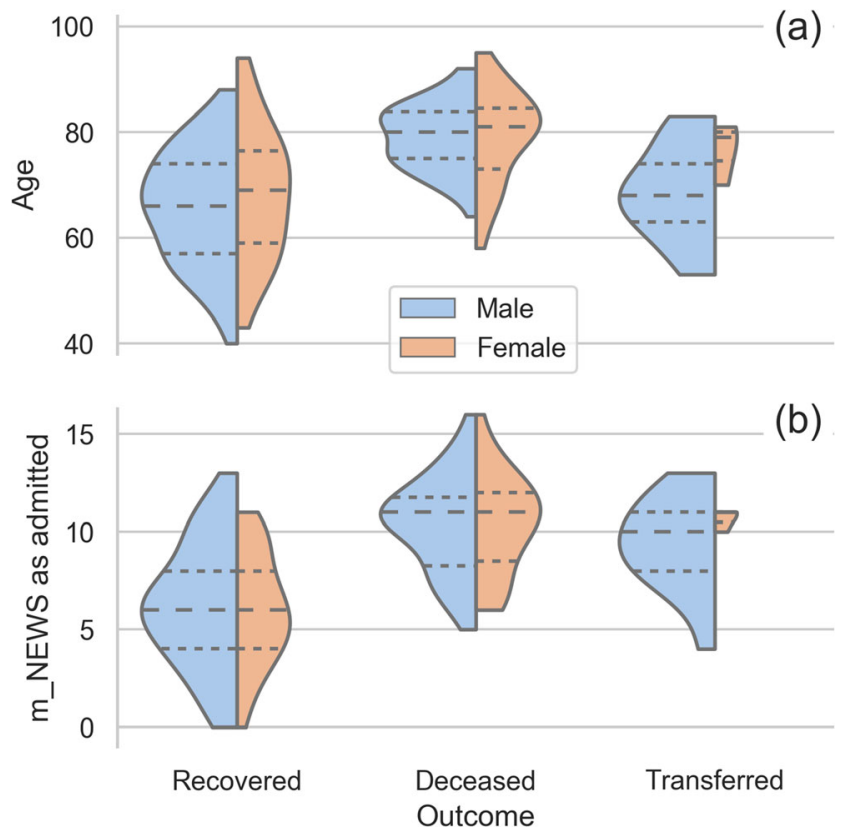

Fig. 1 Violin plot: summary of the probability density estimates of the variables age (a) and m-NEWS at admission (b) in our dataset 2e), that may be attributed to the effects of the immediate treatment administered to the patients.

To compare the performance of age and m-NEWS at admission variables as binary classifiers of the "Recovered" outcome, we plotted their receiver operating characteristics (ROC) in Fig. 3. In the latter, continuous lines represent the characteristics yielded by kernel density estimates, while discrete observations are superimposed as dots. The visible distance between the curves, along with area-under-curve (AUC) values (included in the plot legend), indicates that, in our statistical sample, the m-NEWS at admission vastly outperforms age as a classifier for the "Recovered" outcome. This corroborates the choice of m-NEWS variable as the core of a triage protocol. As an example, a m-NEWS $=7$ cutoff would here be associated with a true positive rate of $60 \%$, and only $10 \%$ false positive rate. The same false positive rate requires an age threshold of 65 years that would however grant only $45 \%$ true positive rate for the "Recovered" outcome.

\section{Discussion}

At the beginning of COVID-19 pandemic, the first clinical observations reported by Chinese colleagues seemed to suggest that mostly of COVID-19 victims were the elderly [23, 24]. As a matter of fact, age on its own showed to have a decisive influence on the patients' outcome [13]. For this reason, we decided to implement, for the first time in our hospital, m-NEWS score that compounds the age parameter with the other relevant physiological factors of NEWS. We compared the triage performance of m-NEWS with respect to the age variable alone. Our results, quantitatively derived from the evaluation of receiver-operating characteristics, show that $\mathrm{m}$ NEWS outperforms age as a classifier of patients' outcome.

We have considered the fact that to develop the "ideal" scoring strategy for COVID-19 patients, it would have been necessary to evaluate every single physiological and epidemiological data item in a univariate and then multivariate logistic regression analysis. This could then lead us to restrict our attention only to variables which would result significantly correlated with the primary outcome (weighted by their odds radio (OR)), but this could not be our aim. Our primary objective was to adopt an existent score (already validated in critical settings) that could give us a satisfactory patient assessment in a timely fashion. The latter requirement was essential for correct patient management, especially in the context of strenuously depleted resources during the pandemic.

$\mathrm{m}$-NEWS showed to be a useful and sensible parameter to detect early patients deterioration in a variety of medical conditions [25]. Concerns regarding its reliability were spotted when applied to patients with chronic low $\mathrm{SpO}_{2}$ saturation level (such as COPD patients) or for every condition that may affect the correct functioning of the autonomic central 

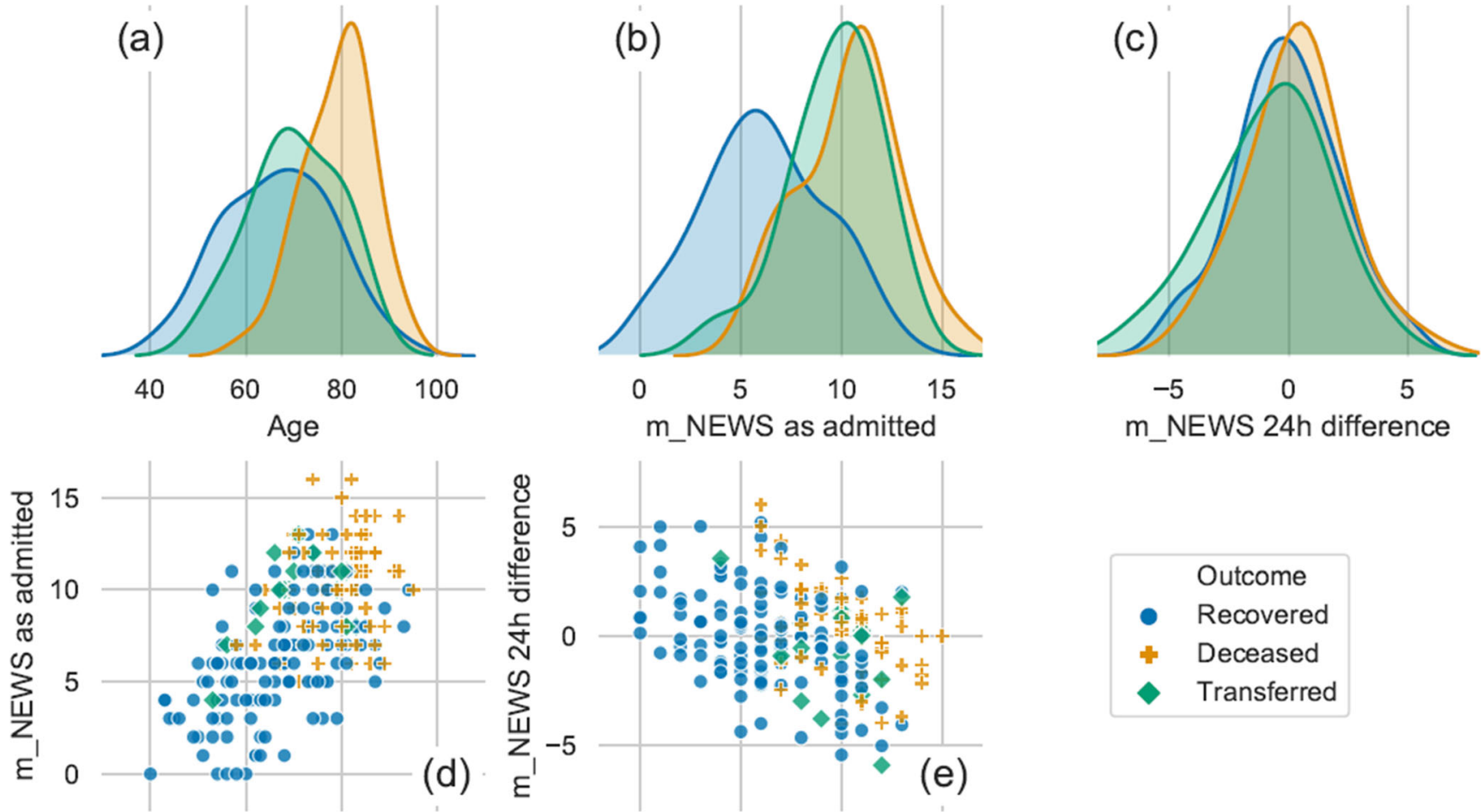

Fig. 2 Variable distribution and cross-correlation in our statistical sample, classified by clinical outcome

nervous system (as in case of tetraplegia or spinal cord damage) that can lead to dysregulation in heart pulse rate, respiratory rate, or temperature [26].

Despite these drawbacks, these scores can be adopted as a common and standardized language to rapidly describe patients' clinical conditions among different healthcare professionals (in particular non-clinicians), residents, and general

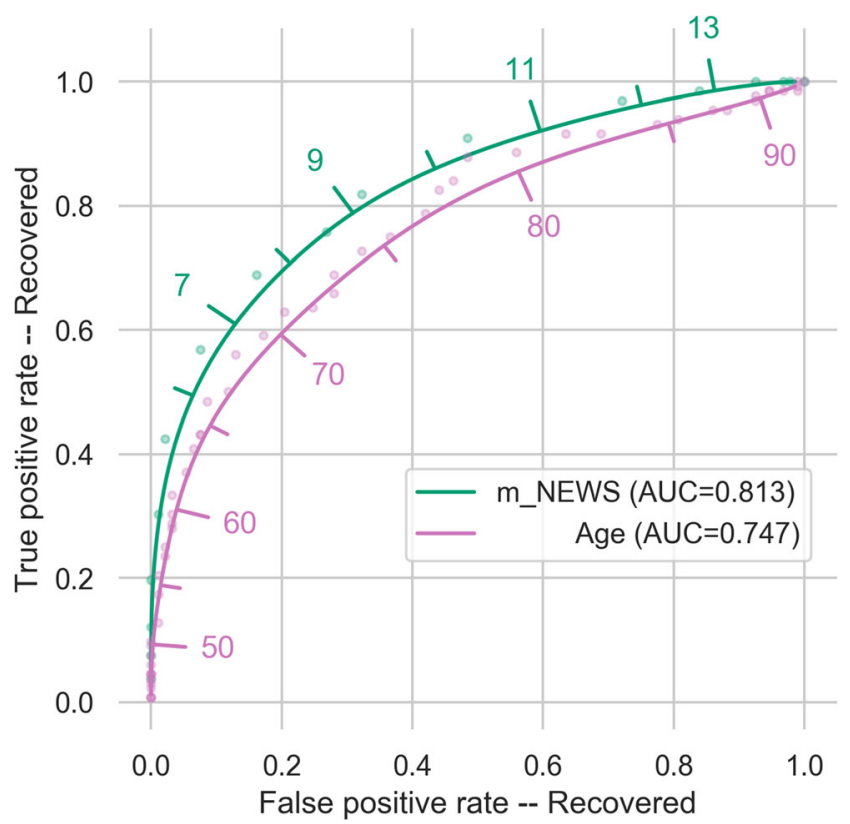

Fig. 3 Receiver operating characteristics (ROC) to compare the performance of age and $\mathrm{m}-\mathrm{NEWS}$ at admission variables practitioners. Their rapidity and easy-to-use described in literature can adapt perfectly to an emergency situation. Calculation of the score is not time-consuming, due to the fact that it is derived from the vital signs that are routinely measured by clinicians and nurses. An interdisciplinary, common, and universal scoring scale to classify the risk of clinical evolution is a valid help in managing a large volume of patients as it happened in the COVID-19 epidemic.

The current indications reported that, in a state of reduced availability of ICU beds, the decision whether to transfer a serious patient or not had to be based more on age than on NEWS [27]. However, this work shows that, in COVID-19 infection, m-NEWS at admission is preferable to age as a classifier for the "Recovered" result. This would indicate the preferability of the variable m-NEWS as the core of protocols for both triaging and escalation to ICU transfer, within our sample.

Despite the clear advantages in terms of clinical evolution with more intensive treatment for patients with $\mathrm{m}-\mathrm{NEWS} \geq 7$ on first evaluation, there is still no consensus for treatment of population with an $\mathrm{m}-\mathrm{NEWS}<7$ on admission. For lower $\mathrm{m}-$ NEWS values, there is not sufficient evidence to strictly recommend a unique therapeutic path.

That being said, our results envision a strategy where, e.g., crossing a specific m-NEWS threshold value would direct hospital physicians to proceed with more intensive treatment (i.e., C-PAP, IV nutrition, and morphine administration) and possible orotracheal intubation.

Naturally, despite m-NEWS reliability and easy-to-use, it has to be considered only a supporting "tool" for a physician's 
or nurse's clinical judgement. In any case, a patient's clinical examination, including anamnesis and comorbidities, has to guide and dictate each treatment path during hospitalization.

\section{Study Limitations}

Our study was designed and executed in an emergency setting. Our "off-label" application of the m-NEWS score has to be considered only in this particular situation, characterized by an explosive number of infected patients that were exerting an incredible pressure on emergency departments [3].

Furthermore, our statistical sample has been characterized by a certain degree of heterogeneity making it questionable to compute a comprehensive statistical multivariate analysis; moreover, limited availability of therapeutic resources renders several statistical observations non-independent within the sample. It is also important to acknowledge that our study represents the particular situation related to Alzano Lombardo territory and it cannot be considered representative of other settings, Italian or otherwise.

Nevertheless, this work is of strong relevance: in view, e.g., of the recurrence of the novel coronavirus outbreak in the first months of 2021, which has tallied (at the time of writing) a total of 2,955,434 recorded cases and 98,288 deaths in Italy alone [28].

\section{Conclusions}

m-NEWS showed to be a reliable and easy-to-use score for first patient evaluation. Its use could aid the patients' management before and during hospitalization. We find that a mNEWS that is 7 or greater at the first patient's evaluation is associated with a negative outcome, indicating a clinical status worth of a more intensive treatment, in retrospect. On the other hand, patients with m-NEWS on the first evaluation under 7 are associated with higher possibility of recovery. Naturally, in order to guarantee the best medical treatment for patients, it is fundamental to interpret the mere score within the full clinical context of the patient. More evidence is needed to verify if a threshold of m-NEWS acquired on the territory (e.g., from general practitioners) can be used as a deciding factor for early hospitalization or domiciliar treatment. Our work invites further proof gathering in order to quantitatively predict optimal threshold values to be used in a more general setting than the emergency considered here.

Authors' Contributions All listed authors made substantial contributions to conception and design, acquisition of data, and analysis and interpretation of data; moreover, all authors participated in drafting the article or revising it critically for important intellectual content and gave final approval of the version to be submitted and any revised version. The corresponding author attests that all listed authors meet authorship criteria and that no others meeting the criteria have been omitted. Fabio Tagliabue and Daniele Schena equally contributed as first authors.

Funding No funding was obtained for the drafting of this manuscript

Data Availability The data that support the findings of this study are available from the corresponding author L.G. upon reasonable request.

Code Availability The code that support the findings of this study are available at https://zenodo.org/record/3767070\#.X5727IhKjIU.

\section{Declarations}

Ethics Approval The study obtained the approval of our local ethical committee, ASST Papa Giovanni XIII, Piazza OMS, 1, 24127 Bergamo, Italy, with resolution no. 302/20 on the 10th of February, 2020.

Consent to Participate The informed consent process was waived due to the observative retrospective nature of the study. To protect confidentiality, data were recorded on an encrypted file and all medical records were replaced with a unique study number. All data were reported in a deidentified and aggregated form and can be accessed only by medical and nursing staff only upon entering a personal password. A substitutive declaration from our local committee will be attached to the present study as "supplementary materials."

Consent for Publication The corresponding author does grant on behalf of all authors. This manuscript has been read and approved for submission to SN Comprehensive Clinical Medicine Journal by all the named authors that participated in the entire content, conception, drafting, and final critical revision of the manuscript.

Conflict of Interest All authors declare that no support from any organization for the submitted work and no financial relationships with any organizations that might have an interest in the submitted work in the previous three years have been received. No other relationships or activities could appear to have influenced the submitted work.

\section{References}

1. Regione Lombardia Emergenza Covid-19 - Avviso pubblico per manifestazione di interesse - Medici specialisti, medici specializzandi e medici laureati (2020) https://www.regione. lombardia.it/wps/portal/istituzionale/HP/DettaglioBando/servizie-\%0Ainformazioni/enti-e-operatori/sistema-welfare/avvisomedici-specializzandi/avviso-medici-\%0Aspecializzandi. Accessed 03 march 2021

2. Galassi L, Schena D. The modified National Early Warning Score (m-NEWS) for COVID-19-infected patient evaluation: a proof-ofconcept. SN Comprehensive Clinical Medicine. 2020;3:9-10. https://doi.org/10.1007/s42399-020-00718-x.

3. Mitchell R, Banks C. Emergency departments and the COVID-19 pandemic: making the most of limited resources. Emerg Med J. 2020. https://doi.org/10.1136/emermed-2020-209660.

4. Semeraro F, Scquizzato T, Scapigliati A, Ristagno G, Gamberini L, Tartaglione M, et al. New Early Warning Score: off-label approach for Covid-19 outbreak patient deterioration in the community. Resuscitation. 2020;151:24-5. https://doi.org/10.1016/j. resuscitation.2020.04.018.

5. Romero-Brufau S, Huddleston JM, Naessens JM, Johnson MG, Hickman J, Morlan BW, et al. Widely used track and trigger scores: 
are they ready for automation in practice? Resuscitation. 2014;85: 549-52. https://doi.org/10.1016/j.resuscitation.2013.12.017.

6. Morgan R, Williams F, Wright M. An early warning scoring system for detecting developing critical illness. Clin Intensive Care. 1997.

7. Pimentel MAF, Redfern OC, Gerry S, Collins GS, Malycha J, Prytherch D, et al. A comparison of the ability of the National Early Warning Score and the National Early Warning Score 2 to identify patients at risk of in-hospital mortality: a multi-centre database study. Resuscitation. 2019;134:147-56. https://doi.org/10. 1016/j.resuscitation.2018.09.026.

8. Mellhammar L, Tverring C, Boyd S, Åkesson K. NEWS2 is superior to qSOFA in detecting sepsis with organ dysfunction in the emergency department. J Clin Med. 2019;8. https://doi.org/10. 3390/jcm8081128.

9. Wattanasit P, Khwannimit B. Comparison the accuracy of early warning scores with qSOFA and SIRS for predicting sepsis in the emergency department. Am J Emerg Med. 2020. https://doi.org/10. 1016/j.ajem.2020.07.077.

10. Patterson C, Maclean F, Bell C, Mukherjee E, Bryan L, Woodcock $\mathrm{T}$, et al. Early warning systems in the UK: variation in content and implementation strategy has implications for a NHS early warning system. Clin Med J R Coll Physicians London. 2011;11:424-7. https://doi.org/10.7861/clinmedicine.11-5-424.

11. Liao X, Wang B, Kang Y. Novel coronavirus infection during the 2019-2020 epidemic: preparing intensive care units - the experience in Sichuan Province. China Intensive Care Med. 2020;46: 357-60. https://doi.org/10.1007/s00134-020-05954-2.

12. Yang X, Yu Y, Xu J, Shu H, Xia J', Liu H, et al. Clinical course and outcomes of critically ill patients with SARS-CoV-2 pneumonia in Wuhan, China: a single-centered, retrospective, observational study. Lancet Respir Med. 2020;8:475-81. https://doi.org/10. 1016/S2213-2600(20)30079-5.

13. Mallapaty S. The coronavirus is most deadly if you are older and male - new data reveal the risks. Nature. 2020;585:16-7.

14. Martinez L, Cheng W, Wang X, Ling F, Mu L, Li C, et al. A risk classification model to predict mortality among laboratoryconfirmed avian influenza A H7N9 patients: a population-based observational cohort study. J Infect Dis. 2019;220:1780-9. https:// doi.org/10.1093/infdis/jiz328.

15. Società Italiana Anestesia Analgesia Rianimazione e Terapia Intensiva (SIAARTI). PERCORSO ASSISTENZIALE PER IL PAZIENTE AFFETTO DA COVID-19 (2021) http://www. siaarti.it/SiteAssets/News/COVID19 - documenti SIAARTI/ Percorso COVID-19 - Sezione 1 - Procedura Area Critica.pdf. Accessed 03 march

16. Società Italiana di Malattie Infettive e Tropicali. Vademecum per la cura delle persone con malattia da COVI-19 (2021) https://www.
sicp.it/wp-content/uploads/2020/04/SIMIT_Vademecum-cura-pzCovid19_13-mar-2020.pdf. Accessed 03 march

17. McKinney W. Data structures for statistical computing in Python. Proc 9th Python Sci Conf. 2010. https://doi.org/10.25080/majora92bf1922-00a.

18. Hunter JD. Matplotlib: a 2D graphics environment. Comput Sci Eng. 2007;9:90-5. https://doi.org/10.1109/MCSE.2007.55.

19. Van Der Walt S, Colbert SC, Varoquaux G. The NumPy array: a structure for efficient numerical computation. Comput Sci Eng. 2011;13:22-30. https://doi.org/10.1109/MCSE.2011.37.

20. Virtanen P, Gommers R, Oliphant TE, et al. SciPy 1.0: fundamental algorithms for scientific computing in Python. Nat Methods. 2020;17:261-72. https://doi.org/10.1038/s41592-019-0686-2.

21. https://zenodo.org/record/3767070\#.X5727IhKjIU.

22. April S. Density estimation for statistics and data analysis chapter 1 and 2. Most. 2003.

23. Mueller AL, Mcnamara MS, Sinclair DA. Why does COVID-19 disproportionately affect older people? Aging (Albany NY). 2020. https://doi.org/10.18632/aging.103344.

24. Starke KR, Petereit-Haack G, Schubert M, Kämpf D, Schliebner A, Hegewald J, et al. The age-related risk of severe outcomes due to covid-19 infection: a rapid review, meta-analysis, and meta-regression. Int J Environ Res Public Health. 2020;17. https://doi.org/10. 3390/ijerph17165974.

25. Gerry S, Bonnici T, Birks J, Kirtley S, Virdee PS, Watkinson PJ, et al. Early warning scores for detecting deterioration in adult hospital patients: systematic review and critical appraisal of methodology. BMJ. 2020. https://doi.org/10.1136/bmj.m1501.

26. Asafu-Adjaye K, Gall A. Letter to the Royal College of Physicians regarding the suitability of the National Early Warning Score in the assessment of the unwell spinal cord injury patient. Clin Med J R Coll Physicians London. 2015;15:406-7. https://doi.org/10.7861/ clinmedicine.15-4-406.

27. RACCOMANDAZIONI DI ETICA CLINICA PER L'AMMISSIONE A TRATTAMENTI INTENSIVI E PER LA LORO SOSPENSIONE, IN CONDIZIONI ECCEZIONALI DI SQUILIBRIO TRA NECESSITÀ E RISORSE DISPONIBILI (2020) http://www.siaarti.it/SiteAssets/News/COVID19documentiSIAARTI/SIAARTI-Covid19-Raccomanda zionidieticaclinica.pdf Accessed 03 march 2021

28. WHO health emergency dashboard COVID-19 in Italy. https:// covid19.who.int/region/euro/country/it. Accessed 03 march 2021

Publisher's Note Springer Nature remains neutral with regard to jurisdictional claims in published maps and institutional affiliations. 